\title{
EFFECT OF A "B" CHROMOSOME ON CHIASMA LOCALISATION AND FREQUENCY IN MALE EUTHYSTIRA BRACHYPTERA
}

\author{
HUGH L. FLETCHER and G. M. HEWITT \\ School of Biological Sciences, University of East Anglia, University Plain, Norwich NR4 7TJ
}

Received 23.x.79

\begin{abstract}
SUMmary
Euthystira brachyptera is one of several species of grasshopper noted for terminal localisation of chiasmata in the males. Chiasma frequency and distribution have been studied in samples collected in the French Alps in 1974 and 1978. Localisation was found to be unusual because two chiasmata occasionally occurred close together in the terminal region of the long bivalents. Chiasmata were not localised in the short bivalents. Some individuals were found to carry a supernumerary " $B$ " chromosome (mean frequency 0.5 (1975) to 0.3 (1978) B's per individual) which apparently reduced localisation while raising the mean cell chiasma frequency and variation from 14.0 (s.e.m. 0.25 ) to 15.1 (s.e.m. 0.71 ). Large variations in chiasma frequency were found between samples collected from neighbouring sites $(50 \mathrm{~m}$ to $300 \mathrm{~m}$ apart) show. ing that the population was very heterogeneous. While this makes it difficult to interpret the results simply, it suggests that chiasma frequency (and hence recombination) is not subject to strong standardising selection.
\end{abstract}

\section{INTRODUCTION}

THE occurrence of supernumerary or " B " chromosomes in Orthopterans is fairly common (see Hewitt, 1973, for review). In general they are accumulated by non-random segregation in germ line mitosis or at meiosis and limited in frequency by their deleterious effects, particularly at high frequencies. "B" chromosomes often raise the chiasma frequencies in autosomal bivalents and increase the variance in chiasma frequencies between cells. The way in which a " $\mathrm{B}$ " chromosome affects autosomes is unknown. Besides its physical presence in a nucleus, it can affect the chemical composition of the nucleoplasm (see Jones, 1975, for review). Euthystira brachyptera is a species of grasshopper noted for terminal localisation of chiasmata in the males (Craig-Cameron and Jones, 1970). A population has been discovered in which many individuals carry a "B" chromosome and this has been used to study the mechanism by which " B" chromosomes affect the autosomes, particularly in the formation and localisation of chiasmata.

\section{Materials AND MEthods}

An initial sample of 16 pairs of testes of Euthystira brachyptera were collected by Dr and Mrs Peter Robinson in 1974, and a further collection of 47 pairs of testes by H. L. F. in 1978. The original sample came from Haute Savoie, France, from a meadow north of the river l'Arve, south of the D13 and west of Vieux Servoz (site 1). In 1978, samples were collected from subpopulations at the high and low ends $(\mathrm{H}$ and $\mathrm{L})$ of this meadow, at the high and low ends of the next meadow to the west (Site 2) and from a clear- 
ing north of the D13 (site 3 ). Sites 1 and 2 were separated by a thin strip of woodland. All the sites were within a $350 \mathrm{~m}$ diameter circle. One half of each pair of testes was fixed in $3: 1$ ethanol acetic acid and standard squash preparations were made in 45 per cent lactopropionic orcein.

Chiasma position was scored by eye in all scorable cells from late diplotene to metaphase. Since most chiasmata were terminal this was simple. The principal criterion for calling a chiasma terminal was that no part of a chromosome arm was visible beyond it at metaphase except for the centromere (and attached heterochromatic knob on the M4 and M5 telocentrics). In extended diplotene chromosomes this was necessarily relaxed to include at most the terminal 20 per cent, but this situation arose in only 0.5 per cent of the cells scored and is negligible. Chiasma which were not at one end of the bivalent arm were either near the middle, or between the middle and one end. Thus the chromosome arm was easily divided into five parts even in condensed metaphase chromosomes where the arms of the long and medium bivalents still exceeded $10 \mu$ in length. The greatest problem was the occurrence of two chiasmata close to the end of the bivalent. The combination could have been scored as one subterminal chiasma. When there were more than three chiasmata in one arm it was difficult to be sure of the actual number present, but such occasions were very rare. When the chromosomes were extended at diplotene, the centromeres of the metacentrics were visible as extended regions showing little staining. The individual bivalents and arms were identified by eye by relative size when necessary, but because similar bivalents in any cell often had similar chiasma distributions, it was not always required.

\section{Results \\ (i) Karyotype}

The B chromosome was metacentric and about the same size as the M4 and M5 (long teleocentrics, fig. la, b, c). It was characteristically folded into a tight ring throughout first meiotic prophase (fig. Id and e). The autosomal complement consists of three long metacentric pairs and two medium length telocentric pairs, all of which have proterminally localised chiasmata in the males, and three short telocentric pairs without localised chiasmata. The telocentric X univalent is peculiar in that in most individuals it becomes elongated and diffuse at metaphase and shows neocentric activity. The $\mathrm{X}$ and $\mathrm{B}$ were both condensed during first meiotic prophase (fig. le).

\section{(ii) Frequency of the $B$ chromosome}

Eight of the original 16 males each had one B chromosome (freq. 0.5 ) but in the second sample of 47 males there was one $2 \mathrm{~B}$, twelve $1 \mathrm{~B}$, and thirtyfour $\mathrm{OB}$ individuals (frequency $0.3 \mathrm{Bs}$ per individual). The apparent drop in frequency has a 7.3 per cent probability $\left(\chi_{(1)}^{2}=3.218\right)$ of occurring by chance variation, but may be real.

\section{(iii) Chiasma frequency}

Mean chiasma frequencies per cell for each subsample are shown in table 1 . In the original 1974 sample there was significant variation in 
TABLE 1

Cell mean chiasma frequencies in male Euthystira brachyptera without $B$ chromosomes $(O B)$ and with B chromosomes $(1 B)$ collected at neighbouring sites in 1974 and 1978

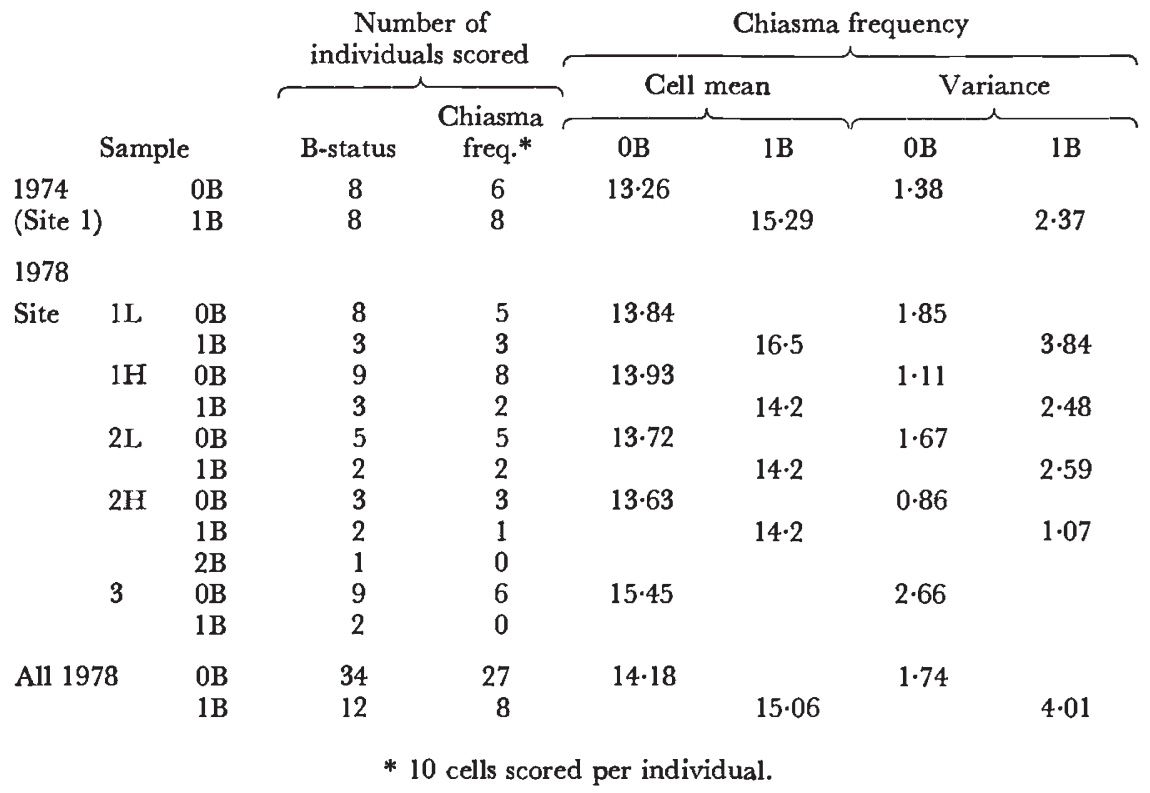

chiasma frequency between individuals within each group $\left(0 \mathrm{~B}, \mathrm{~F}_{(5,52)}=\right.$ $\left.2.52, \mathrm{P}<0.05 ; 1 \mathrm{~B}, \mathrm{~F}_{(5,59)}=12.2, \mathrm{P}<0.001\right)$. Similarly high variance between individuals was found in the 1978 samples (table 2). The presence of a B chromosome in members of the 1974 sample caused a significant rise in cell mean chiasma frequencies $\left(\mathrm{t}_{125}=5.7, \mathrm{P}<0.001\right)$ and an increase in variance between cells $\left(\mathrm{F}_{(68,57)}=2 \cdot 936, \mathrm{P}<0.001\right)$ and individuals $\left(\mathrm{F}_{(7,5)}=\right.$ $7 \cdot 015, \mathrm{P}<0.025$ ).

In the second sample, substantial differences were found from the first group. Only individuals where more than 10 cells were scored are included in the analysis. The variation between individuals made it difficult to compare the five subpopulations. Some individuals had nearly perfect chiasma localisation (two terminal chiasmata per metacentric) while others also had interstitial chiasmata in many cells. Two subsamples had anomalously high chiasma frequencies: three $1 \mathrm{~B}$ individuals from site $1 \mathrm{~L}$ (cell mean $16 \cdot 5$, s.d. 1.96) and six 0B males from site 3 (cell mean $15 \cdot 45$, s.d. 1.63 ).

TABLE 2

Analysis of variance of chiasma frequency in male Euthystira brachyptera without $B$ chromosomes $(O B)$ collected in 1978

$\begin{array}{lccccc}\quad \text { Source } & \begin{array}{c}\text { Degrees of } \\ \text { freedom }\end{array} & \begin{array}{c}\text { Sum of } \\ \text { squares }\end{array} & \begin{array}{c}\text { Mean } \\ \text { square }\end{array} & \begin{array}{c}\text { Variance } \\ \text { ratio }\end{array} & \text { Probability } \\ \text { Subsamples } & 4 & 127 \cdot 3 & 31 \cdot 825 & 3 \cdot 16 & \mathrm{P}<0.05 \\ \text { Individuals } & 27 & 263 \cdot 066 & 9 \cdot 743 & 29.20(27,237) & \mathrm{P}<0.01 \\ \text { Error } & 237 & 79 \cdot 099 & 0.333 & & \\ \text { Total } & 269 & 469 \cdot 466 & 1.745 & & \\ \quad 44 / 3-D & & & & & \end{array}$


An analysis of variance of all the second sample $O B$ individuals (table 2) shows a highly significant variance ratio between individuals $(P<0.01)$ and also between sites $(P<0 \cdot 05)$. This could be due to heterogeneity in the genetic or environmental background, particularly visible at site three. The former could be produced by genetic drift in an inbred group of individuals of a sedentary species, which is what Euthystira appears to be. The environment did not appear to vary markedly, although site $1 \mathrm{~L}$ was the lowest, coolest and grassiest site and 3 was highest, with most herbs. Either cause suggests that recombination rate is labile and easily varied. The results were pooled into $\mathrm{a} \mathrm{OB}$ and $\mathrm{IB}$ group to reduce sampling error and again the $1 \mathrm{Bs}$ had higher mean chiasma frequency and variance (1B mean 15.06, s.d. $2.003 ; 0 B$ mean $14 \cdot 18$, s.d. $1.321 ; \mathrm{t}_{348}=4.609, \mathrm{P}<0.001 ; \mathrm{F}_{(80,270)}=$ $2 \cdot 229, \mathrm{P}<0.001)$. The 1978 OB group also had a higher cell chiasma frequency than the $19740 \mathrm{Bs}\left(\mathrm{t}_{\mathbf{3 6 2}}=3.971, \mathrm{P}<0.001\right)$.

\section{(iv) Chiasma distribution}

The positions of chiasmata were scored by eye, a process considerably helped by the strong terminal localisation found in most bivalents. The approximate divisions used (five parts per long and medium chromosome arm, three parts per short chromosome) and the corresponding frequency of chiasmata in each class is shown in fig. 3. The largest error is likely to be in the density of terminal chiasmata. Apart from procentric "terminal" chiasmata on the M4 and M5, where the centromeric knob was always separated from the chiasmata by a thin thread of chromatin, no free ends of chromosomes were visible beyond chiasmata scored as terminal. Thus it is probable that they occupied much less than the 20 per cent of the chromosome arms allocated in the histograms, so the terminal bars could be thinner and correspondingly taller. The presence of a $\mathrm{B}$ chromosome (broken lines) tended to increase the frequency of chiasmata at the interstitial and subterminal positions thereby reducing the localisation. This effect was only shown in the long metacentrics and principally in the Ll. The strict localisation in the long telocentrics was unaffected. In the 1974 sample the proportion of terminal chiasmata (a measure of localisation) was much higher in the $\mathrm{OB}$ individuals than in the $1 \mathrm{Bs}$. In the 1978 sample the proportion of terminal chiasmata was not significantly higher in the $O B$ individuals but the $1974 \mathrm{OBs}$ had a higher proportion of terminal chiasmata than the $1978 \mathrm{OBs}$, reflecting their respective chiasma frequencies. Lower chiasma frequencies correspond to stricter localisation. The variation between years is similar to the difference between $\mathrm{OB}$ and $\mathrm{IB}$ individuals, and in the larger (1978) study individual animals tended to have either strictly localised chiasmata or several interstitial chiasmata, irrespective of the presence of a B. The only safe conclusion, therefore, is that chiasmata tend to be more frequent and less localised in individuals with a $\mathrm{B}$ chromosome than in those without one. About half of the subterminal chiasmata in the metacentrics were very close to a terminal chiasmata (e.g., fig. ld) indicating a very short absolute interference distance, although such terminal pairs were much less common than expected from random occurrence of both at their respective frequencies $\chi_{1}^{2}=20, \mathrm{P}<0.001$ ) showing that in this situation one chiasmata inhibits, but does not prevent, formation of another beside it. 
Plate I

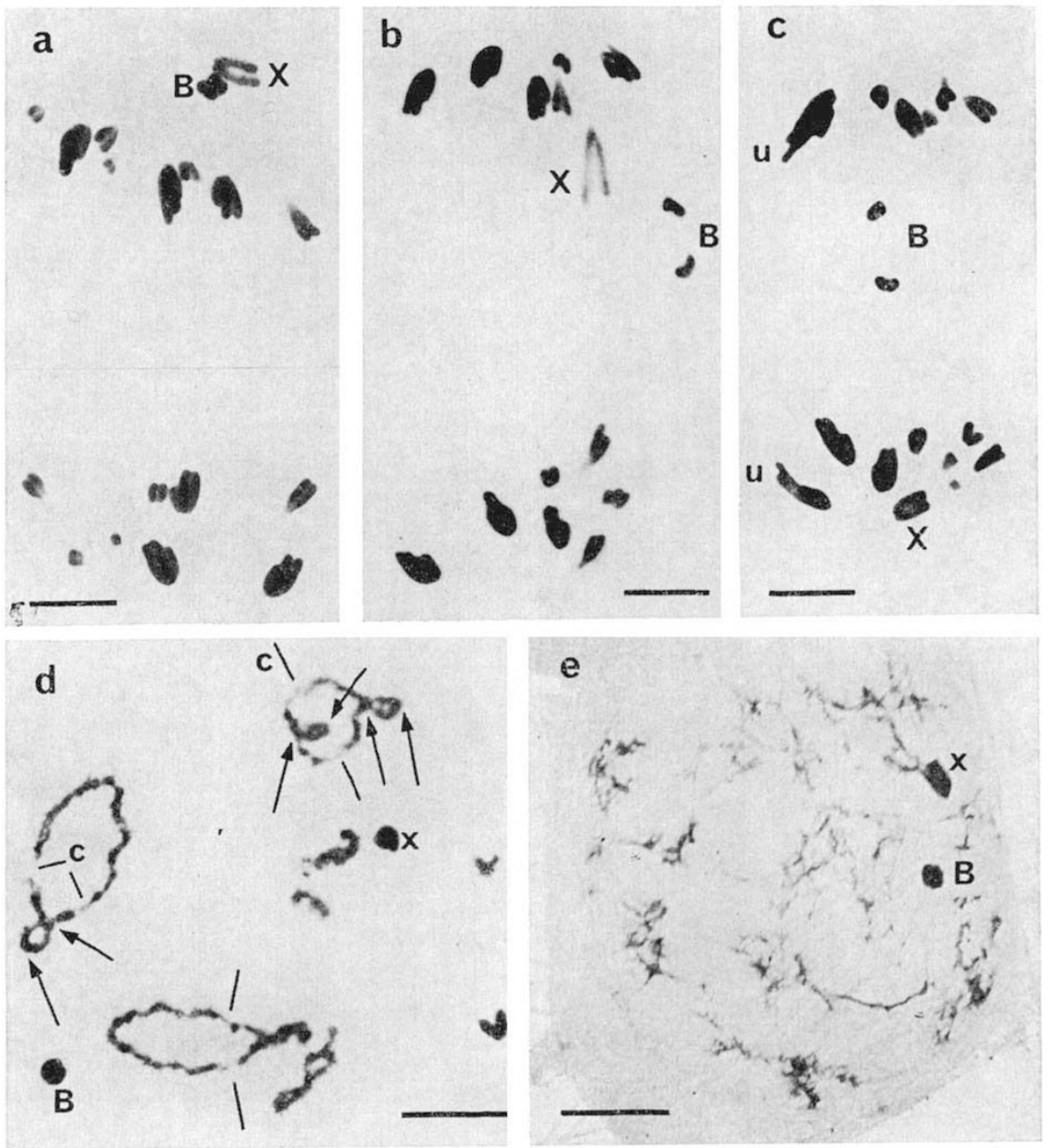

FIG. 1 a-c.-Anaphase I in IB cells, showing the X and B segregating early together (a), and the $B$ dividing at first anaphase accompanied by late (b) and early (c) segregation of the $\mathrm{X}$ univalent. Some of the chromatids in (c) are of unequal length (u) probably because they were stretched by a chiasma. (d) Early diplotene, the first stage at which bivalents were resolvable, showing two chiasmata close together at the ends of three arms of the metacentrics (arrows). Centromeres-c-are shown by lines. Some relic coiling is also visible in these bivalents. (e) Pachytene/diplotene with only the $\mathrm{X}$ and $\mathrm{B}$ univalents resolvable. Bars $=20 \mu$. 
Plate II

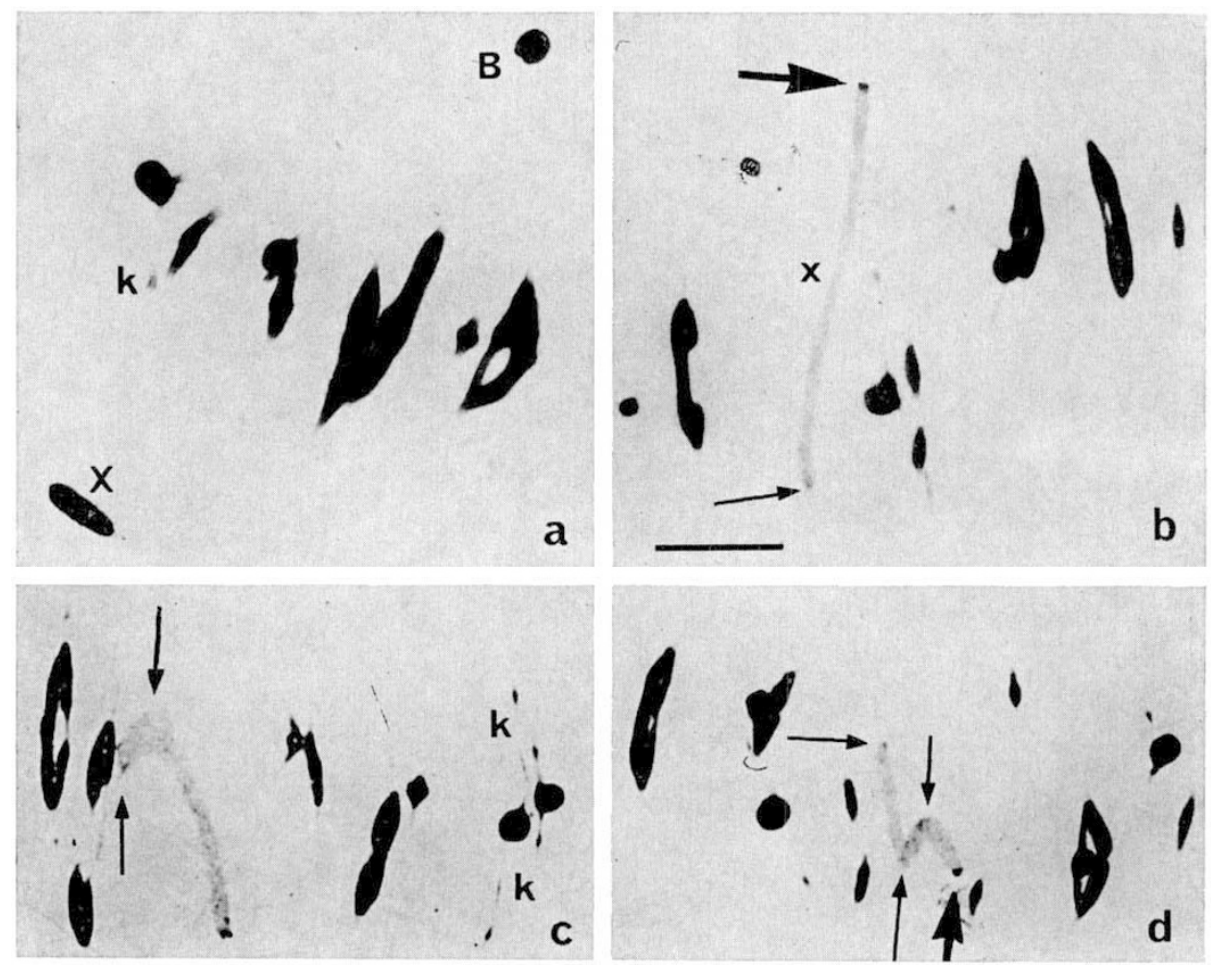

FIG. 2 a-d.-Metaphases showing a condensed $\mathrm{X}$ univalent segregating to the pole opposite the $B$ (a), and diffuse $X$ univalents with one (b), two (c) and three (d) neocentres (small arrows). The true centromeres remain densely staining (large arrows). The centromeric knobs (k) can be seen on the M4 and M5 bivalents in all four cells. Bar $=20 \mu$. 


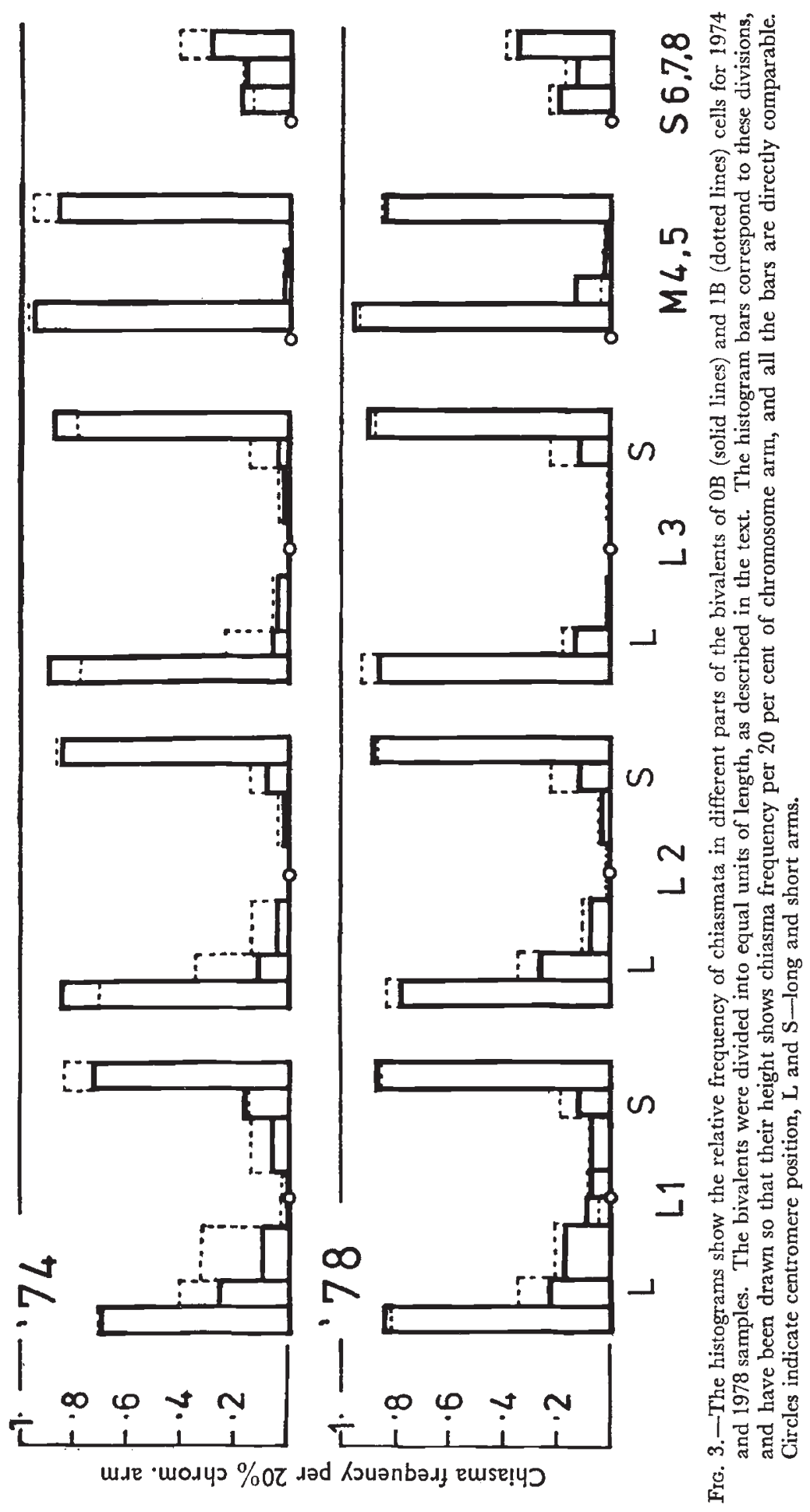


The long telocentrics nearly always had a procentric chiasma, regularly formed rings and rarely had interstitial chiasmata. The three short chromosomes were too short to score accurately, but did not have localised chiasmata. This is similar to the situation found in male Chrysochraon dispar, Parapleurus alliaceus, (Fletcher and Hewitt, 1980) and Stethophyma grossum (Perry and Jones, 1974) which also have localisation in the longest, but not the three shortest, bivalents. Chrysochraon has a very similar karyotype to Euthystira but much stricter localisation of chiasmata which are terminal in both metacentrics and long telocentrics.

\section{(v) Behaviour of the $X$ and $B$ chromosomes at Metaphase I-Anaphase I}

The X univalent usually became decondensed, extended and diffuse at metaphase, in which case it aligned across the metaphase plate. Neocentric activity was apparent at the distal end and often at one or two interstitial sites, so that the $\mathrm{X}$ could stretch almost from pole to pole (fig. $2 \mathrm{~b}$ ) or zigzag between sites where it reacted with the spindle fibres (fig. $2 \mathrm{c}$ and d). The true centromeres remained visible as two adjacent dense dots. In some cells, particularly in some individuals, the $\mathrm{X}$ remained condensed (fig. 2a). It was then likely to segregate prematurely to a pole (about 2.5 to 1 ), as is usual in male Orthoptera. The situation was complicated by intermediate levels of decondensation. A much greater proportion of $\mathrm{Xs}$ was condensed in the $\mathrm{IB}(0.5)$ than the $\mathrm{OB}(0 \cdot 17)$ individuals. On some occasions the $\mathrm{X}$ was seen to lag and segregate after the autosomes at anaphase 1 (fig. $1 \mathrm{~b}$ ).

The $\mathrm{B}$ was always condensed and in a tight ring from the earliest stage of pachytene at which chromosomes became visible (fig. le). Its position was variable in each individual with roughly equal numbers at the equator and the poles. The $\mathrm{X}$ and $\mathrm{B}$ showed a strong tendency for both to stay at the equator, or for both to segregate (apparently independently) to a pole before anaphase (table $3, \chi_{(1)}^{2}=22.03, \mathrm{P}<0.001$ ). This was probably because they were in the same nuclear environment, and again this pattern was subject to drastic variation between individuals. The B was occasionally seen to lag and/or divide at first anaphase (fig. lb and c) but more commonly segregated at anaphase I and divided at anaphase II. All possible modes and combinations of segregation and behaviour of the $\mathrm{X}$ and $\mathrm{B}$ were seen, but were fairly consistent in cells from any one animal.

TABLE 3

Correlation of the position of the $X$ and $B$ chromosomes at metaphase $I$ in 167 cells from 16 male Euthystira brachyptera

\begin{tabular}{|c|c|c|c|c|}
\hline & & \multicolumn{2}{|c|}{ Position of $B$} & \\
\hline & & Equator & Pole & \\
\hline $\begin{array}{l}\text { Position } \\
\text { of } \mathrm{X}\end{array}$ & $\begin{array}{l}\text { Equator } \\
\text { Pole }\end{array}$ & $\begin{array}{l}66 \\
14\end{array}$ & $\begin{array}{l}36 \\
41\end{array}$ & $X_{(\mathbf{1})}^{2}=22.03, P<0.001$ \\
\hline
\end{tabular}

\section{Discussion}

The most surprising thing about these samples was the tremendous variation in chiasma frequency over very short distances. If the cause is genetic it suggests that the grasshoppers are very sedentary and inbred and that there is not much direct selection for recombination rate. In the 1974 
sample there was a sharp distinction between the $\mathrm{OB}$ individuals with their fairly strict localisation of chiasmata, and the 1 Bs with much less localisation. The second, more widespread sample had individuals of both types with strict or poor localisation. In male Chrysochraon, Parapleurus, and Stethophyma, individuals with poor localisation are rare. It will be interesting in future to monitor the frequency of the $\mathrm{B}$, and also to map its presumably limited distribution, since it has not been reported before. The differences between the 1974 and 1978 samples suggest a changing situation which could be related to adaptation to accommodate a newly arisen $\mathrm{B}$ chromosome, but could equally be due to sampling error in the heterogeneous population. The Euthystira B chromosome is very similar in appearance to the B of Myrmeleotettix maculatus (John and Hewitt, 1965) and has a similar effect, increasing the mean and variance of chiasma frequencies. Meiosis in several species is affected by the presence of $\mathbf{B}$ chromosomes (see Hewitt, 1973, for review).

The mechanism(s) of chiasma localisation in Euthystira is not known but does not seem to depend wholly on the failure of pairing of parts of bivalents (Craig-Cameron and Jones, 1970). In the metacentrics it could be due to a failure of, or delay in, pairing in interstitial regions. Virtually all the long telocentric M4 and M5 bivalents had a proximal chiasma and usually a second, distal one. The localisation was consistent and could be readily fitted to the hypothesis of terminal initiation of bivalent synapsis; it is obvious that hypotheses of interference cannot explain the localisation of single chiasmata, nor the first one of a pair. The three short bivalents usually had single, non-localised chiasmata, suggesting that they were fully paired but interference usually extended over the whole bivalent.

Some of the anaphase I chromosomes in one individual showed one chromatid much longer than the other. This is clearly shown in fig. lc. The long chromatid on the longest metacentric is thinner than its shorter sister. This could represent anaphase stretching of the chromatids involved in chiasmata rather than unequal recombination. The asymmetry of the $\mathrm{B}$ in fig. $1 \mathrm{~b}$ and $\mathrm{c}$ suggest that a chiasma might have formed between chromatids in one arm, rather than between opposite arms. The folding of the B into a ring could indicate that it is an isochromosome, i.e., that the arms are mirror images derived by centromeric fusion of sister chromatids (centromeric misdivision). The $\mathrm{B}$ was so condensed that neither pairing nor chiasmata could be seen. If the B is a self-pairing isochromosome it should be possible to identify its synaptonemal complex by electron microscopy. The $\mathrm{X}$ univalent also folded back tightly on itself.

\section{REFERENCES}

GRAIG-GAMERON, T. A., AND jONEs, G. H. 1970. Meiosis in Euthystira brachyptera. Annals Stat. Biologique Besse-en-Chandesse, 5, 275-292.

FLETCHER, H. L., AND HEWITT, G. M. 1980. A comparison of chiasma frequency and distribution between sexes in three species of grasshopper. Chromosoma, 77, 129-144.

HEWIT, G. H. 1973. The integration of supernumerary chromosomes into the grasshopper genome. Cold Spring Harbor Symp. Quant. Biol., 38, 183-194.

JOHN, B. AND HEWITT, G. H. 1965. The B chromosome system of Myrmeleotettix maculatus (Thunb.). I. The mechanics. Chromosoma (Berl.), 16, 548-578.

JONES, R. N. 1975. B-chromosome systems in flowering plants and animal species. Int. Rev. Cytol., 40, 1-100.

PERry, P. E., AND JONEs, G. H. 1974. Male and female meiosis in grasshoppers I. Stethophyma grossum. Chromosoma (Berl.), 47, 227-236. 